\title{
Effect of Tris(trimethylsilyl) Phosphate Additive on the Electro- chemical Performance of Nickel-rich Cathode Materials at High Temperature
}

\author{
Seol Heui Jang ${ }^{1}$, Junyoung Mun ${ }^{2 *}$, Dong-Ku Kang ${ }^{1}$, and Taeeun Yim ${ }^{1 *}$ \\ ${ }^{1}$ Department of Chemistry, Incheon National University, Academy-ro 119, Yeonsu-gu, Incheon 22012, Republic of Korea \\ ${ }^{2}$ Department of Energy and Chemical Engineering, Incheon National University, Academy-ro 119, Yeonsu-gu, Incheon \\ 22012, Republic of Korea
}

\begin{abstract}
$\mathrm{LiNi}_{x} \mathrm{Co}_{y} \mathrm{Mn}_{z} \mathrm{O}_{2}$ cathode materials have been the focus of much attention because of their high specific capacity. However, because of the poor interfacial stability between cathodes and electrolytes, the cycling performance of these materials fades rapidly, especially at high temperatures. In the present paper, we propose the use of tris(trimethylsilyl) phosphate (TMSPO), which contains phosphate and silyl functional groups, as a functional additive in electrolytes. The addition of TMSPO resulted in the formation of cathode electrolyte interphase (CEI) layers on the surfaces of the cathodes and effectively suppressed electrolyte decomposition reactions, even at high temperatures. As a result, cells cycled with TMSPO exhibited remarkable capacity, which remained after 50 cycles $(82.0 \%)$, compared to cells cycled without TMSPO (64.6\%).
\end{abstract}

Keywords : Lithium-ion battery, Electrode, Cathode, Additive, Formation protocol

Received : 22 February 2017, Accepted : 13 May 2017

\section{Introduction}

Li-ion batteries (LIBs) have long been the powerhouses of mobile devices (i.e., cellphones and laptops) because of their stable cycling performance and moderate rate capability [1-3]. In recent years, the energy conversion/storage system abilities of LIBs have become the focus of attention in other areas, such as in electric vehicles and energy storage systems [4-6]. The use of high-energy density LIBs would allow electric vehicles to travel longer distances, and energy storage systems to have longer operating times.

In LIBs, the energy density of the cell is proportional to the specific capacity and working potential of the electrode material. Many attempts have been made to develop advanced electrode materials to enhance the electrochemical performance of LIBs.

*E-mail address: yte0102@inu.ac.kr (T. Yim) and jymun@inu.ac.kr (J. Mun) DOI: https://doi.org/10.5229/JECST.2017.8.2.162
Recently, the potential of a $\mathrm{Ni} / \mathrm{Co} / \mathrm{Mn}$-based layered oxide material $\left(\mathrm{LiNi}_{x} \mathrm{Co}_{y} \mathrm{Mn}_{z} \mathrm{O}_{2}\right)(\mathrm{NCM})$ as an alternative cathode material to conventional $\mathrm{LiCoO}_{2}$ (LCO) was studied because it offers higher specific capacity ( $\geq 175 \mathrm{~mA} \mathrm{~h} \mathrm{~g}^{-1}$ vs. $\sim 150 \mathrm{~mA} \mathrm{~h} \mathrm{~g}^{-1}$ ) [7-12]. However, the cycle life of NCM containing a high Ni content (high-Ni NCM) was poor because of both structural and surface instability. Indeed, the cation mixing that occurs during the exchange of $\mathrm{Li}$ and $\mathrm{Ni}$ (which is attributed to the similar radii of $\mathrm{Li}^{+}$and $\mathrm{Ni}^{2+}$ ) causes structural deterioration, resulting in the fading of high-Ni NCM-based cells [13-15]. Regarding the surface stability, highly reactive $\mathrm{Ni}^{4+}$, which in the charging process, or residual Li species (i.e., $\mathrm{Li}$ carbonate or Li hydroxide) can accelerate the electrochemical decomposition of the electrolyte and lead to surface resistance, which is the main reason for cell failure $[13,16,17]$. Therefore, the interfacial stability of the high-Ni NCM material must be enhanced to achieve high cell performance.

In this work, we propose tris(trimethylsilyl) phos- 

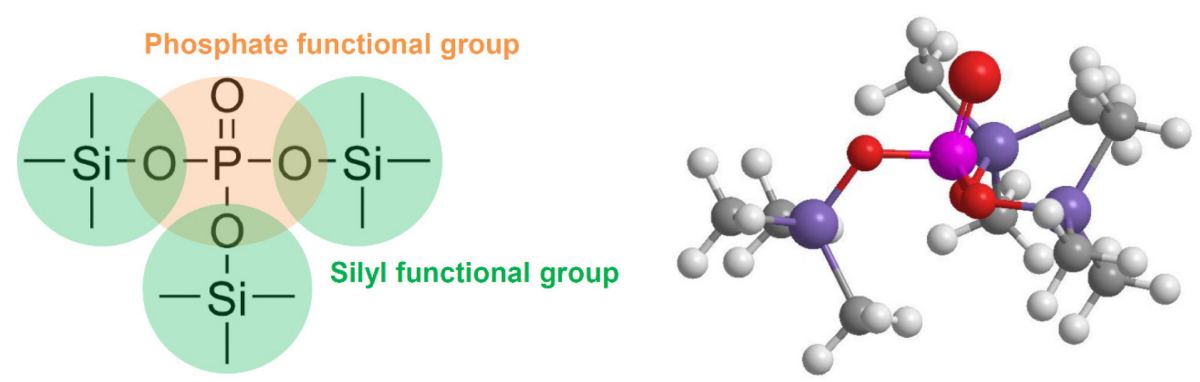

Fig. 1. Two- and three-dimensional molecular structures of TMSPO.

phate (TMSPO) as an effective functional additive to enhance the interfacial stability of high-Ni NCM cathode materials (Fig. 1). TMSPO is distinguished by the presence of two functional groups (phosphate and silyl) in its molecular structure. In previous studies, that tris(trimethylsilyl) phosphite (TMSP), which is composed of phosphite and silyl functional groups, was found to effectively enhance the electrochemical performances of several types of cathode materials because the electrochemical reaction of TMSP produced an effective cathode electrolyte interphase (CEI) layer on the electrode surface [18-23]. In this regard, the use of a phosphate functional group is expected to produce CEI layers on an Ni-rich NCM electrode surface. The reason for this expectation is that the electrochemical reactivity of TMSPO is similar to that of TMSP because the chemical structures of the phosphate and phosphite functional groups are almost identical [24,25]. In addition, the use of the phosphate functional group should be more efficient in terms of water sensitivity because the phosphate functional group is more chemically stable than the phosphite moiety. The role of silyl functional groups in the selective scavenging of fluoride species $\left(\mathrm{F}^{-}\right)$ and, thereby, accelerating the irreversible dissolution of transition metal components in electrolytes is well known [20-22]. Given the aforementioned information, we investigated the utility of TMSPO as a functional additive in a high-Ni NCM material and optimized CEI formation by varying the initializing temperature.

\section{Experimental Section}

For linear sweep voltammetry (LSV), three-electrode beaker cells were fabricated with $\mathrm{Al}$ (for anodic stability) or $\mathrm{Cu}$ (for cathodic stability) as a working electrode and Li metal as both the counter and reference electrodes. The voltage of the cells was swept from 3.0 to $5.0 \mathrm{~V}$ (vs. $\mathrm{Li} / \mathrm{Li}^{+}$) for anodic stability and 3.0 to $0.0 \mathrm{~V}$ (vs. $\mathrm{Li} / \mathrm{Li}^{+}$) for cathodic stability at a rate of $10 \mathrm{mV} \mathrm{s}^{-1}$. A standard electrolyte was used (a combination of ethylene carbonate:ethyl methyl carbonate $\left[1: 2\right.$ by $\left.\mathrm{wt}^{\%}\right]$ ) that contained $1-\mathrm{M} \mathrm{LiPF}_{6}$ (PanaxEtec). TMSPO was purchased from a commercial supplier (Aldrich).

Electrodes were prepared so that their electrochemical performance could be examined. First, an electrode slurry consisting of $90 \% \mathrm{LiNi}_{0.8} \mathrm{Co}_{0.1} \mathrm{Mn}_{0.1} \mathrm{O}_{2}$ (NCM811, Ecopro) as the cathode material, 5\% poly(vinylidene difluoride) as a binder, and 5\% carbon black (super P) as the conductive agent was created. This slurry was then dispersed in $N$-methyl pyrrolidone for $6 \mathrm{~h}$. After the coating process, the electrodes were dried in a vacuum oven at $120^{\circ} \mathrm{C}$ for $12 \mathrm{~h}$. The loading level of the electrodes was fixed at approximately $14.55 \pm 0.36 \mathrm{mg} \mathrm{cm}^{-2}$.

For galvanostatic charge/discharge cycling, 2032 coin-cells were assembled using the NCM811 electrode as a cathode, Li metal as an anode, poly(ethylene) as a separator, and each electrolyte. The cells were cycled at a rate of $0.1 \mathrm{C}$ for two cycles at room temperature (1.0 C $=180 \mathrm{~mA} \mathrm{~g}^{-1}$, formation step). Thereafter, the cells were further cycled at a rate of $1.0 \mathrm{C}$ for 50 cycles at room temperature or $60^{\circ} \mathrm{C}$ (cycling step). The potential ranges of charge/discharge were from 3.0 to $4.3 \mathrm{~V}$ (vs. $\mathrm{Li} / \mathrm{Li}^{+}$).

After completing the cycling tests, the cells were disassembled in an Ar-filled glove box, and each cycled electrode was recovered. The cycled electrodes were quickly washed with dimethyl carbonate and left to dry in the glove box. The surface morphology of the electrodes was then analyzed using scanning electron microscopy (SEM; FEI), and the 

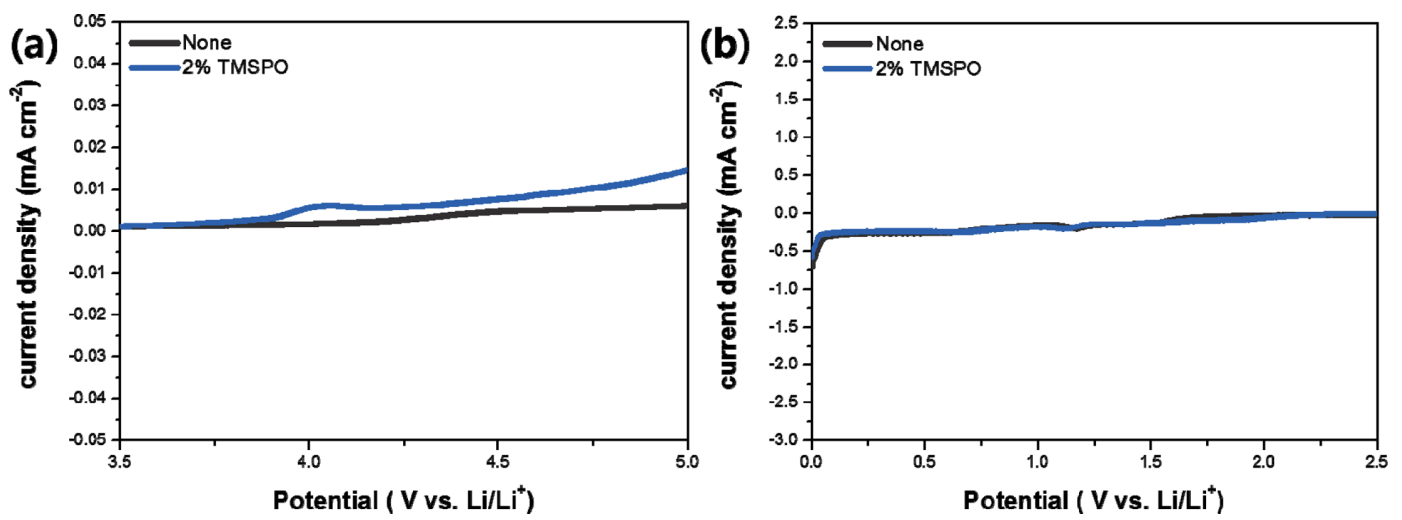

Fig. 2. LSV results for electrolytes at the (a) anodic limit and (b) cathodic limit (black: standard electrolyte; blue: standard electrolyte $+2 \%$ TMSPO).

(a)

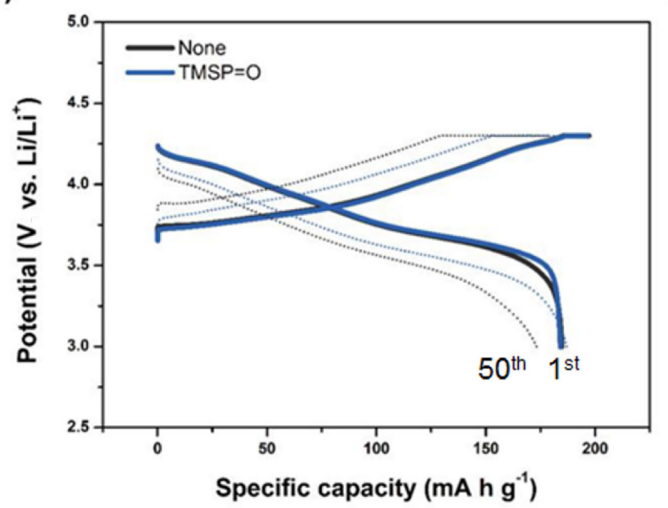

(b)

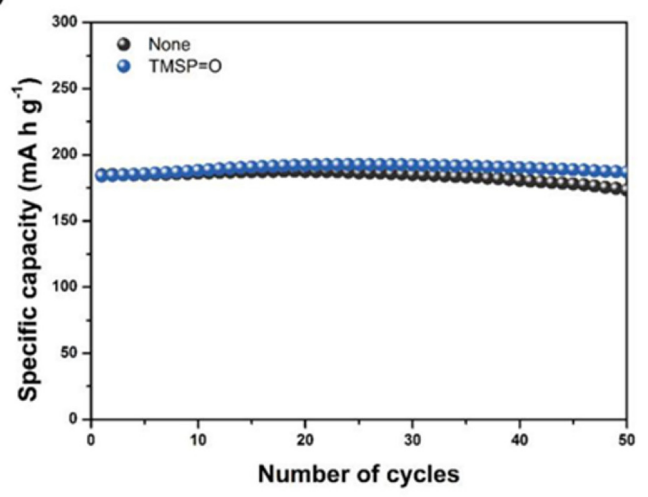

Fig. 3. Electrochemical performances of cells cycled with standard electrolyte (black) and TMSPO-containing electrolyte (blue): (a) potential profiles of the initial cycle (line) and 50 cycles (dot) and (b) cycling retentions. All cycles were performed at room temperature.

chemical components that developed on the cycled electrodes were characterized by Fourier transform infrared spectroscopy (FT-IR, Bruker). Electrochemical impedance spectroscopy (EIS) of the cell charged to $4.3 \mathrm{~V}$ (vs. $\left.\mathrm{Li}^{2} / \mathrm{Li}^{+}\right)$was performed with an $\mathrm{AC}$ signal at an amplitude of $10 \mathrm{mV}$ over a frequency range of $1 \mathrm{M}$ $10 \mathrm{mHz}$ using an electrochemical workstation (Zive MP1, Wonatech).

\section{Results and Discussion}

The electrochemical behavior of TMSPO was characterized via an LSV experiment (Fig. 2). We anticipated that TMSPO would produce CEI layers on the surfaces of the electrodes through an electro- chemical oxidation reaction, and oxidative currents associated with the electrochemical oxidation of TMSPO appeared at approximately $3.8 \mathrm{~V}\left(\mathrm{vs} . \mathrm{Li} / \mathrm{Li}^{+}\right)$ in the electrolyte containing 2\% TMSPO (Fig. 2a). Although this peak does not confirm the effectiveness of the CEI layer, TMSPO clearly changed the surface properties of the NCM811 electrode via an electrochemical oxidation reaction. In the reduction behavior, the electrolyte composed of $2 \%$ TMSPO exhibited electrochemical behaviors that were identical to the standard electrolyte when the potential decreased (Fig. 2b). This result implies that TMSPO would be compatible with conventional carbon-based anode materials. Based on the additive screening results, TMSPO was employed as an additive in cells 
(a)

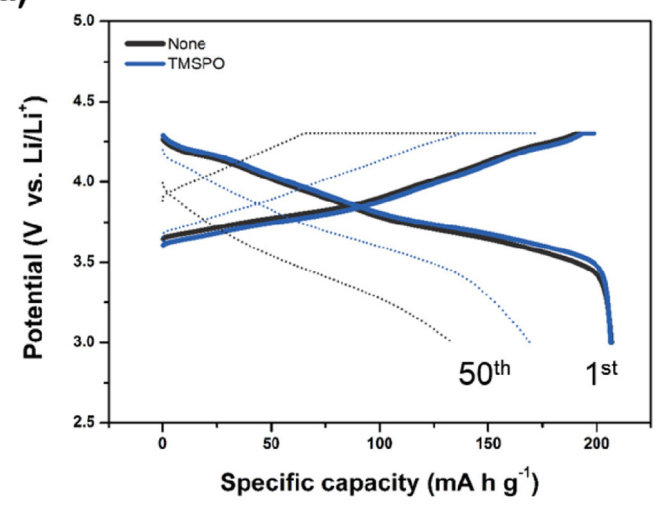

(b)

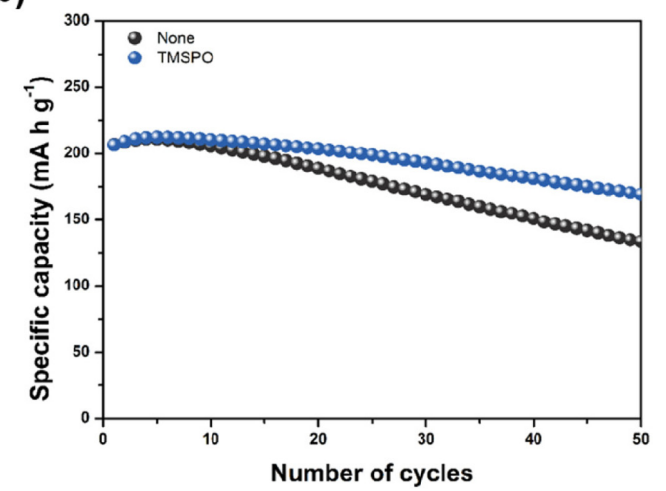

Fig. 4. Electrochemical performances of cells cycled with standard electrolyte (black) and TMSPO-containing electrolyte (blue): (a) potential profiles of the initial cycle (line) and 50 cycles (dot) and (b) cycling retentions. Pre-cycling (i.e., the formation step) was conducted at room temperature, and subsequently, for the cycling test, the temperature was increased to $60^{\circ} \mathrm{C}$.

assembled with the NCM811 cathode to estimate the effect of the electrochemical reaction of TMSPO on the electrochemical performance.

Fig. 3 depicts the cycling performance of the cells at room temperature. Overall, the shapes of the potential profiles of the cells appeared to be identical during the initial cycle, regardless of the inclusion of TMSPO. However, the polarization behaviors varied depending on the use of TMSPO, with the cells cycled with TMSPO exhibiting lower polarization than those cycled without TMSPO (Fig. 3a). As shown in Fig. 2, TMSPO was electrochemically oxidized on the surfaces of the electrodes. The electrochemical reaction of TMSPO might have improved the surface properties of the NCM811 electrode, and the CEI layers derived from TMSPO may have improved the interfacial stability, resulting in lower cell polarization. Overall, the potential profiles of the cells after 50 cycles were very different depending on whether TMSPO was present. As reported previously, the polarization of cells with TMSPO was much better than that of cells without TMSPO because of increased interfacial resistance $[16,17]$.

Cycling retention indicates the effectiveness of TMSPO on the stability of NCM811 cathodes during cycling. In the present study, the cells with TMSPO exhibited almost $100.0 \%$ retention, whereas the cells containing only the standard electrolyte showed continuous fading of retention (93.8\%) after 50 cycles (Fig. 3b). This effect of TMSPO was more evident during high-temperature cycling (Fig. 4). As noted earlier, all the cells were precycled at room temperature to initiate the formation of CEI layers (formation step). Subsequently, the cycling performances of the cells were evaluated at $60^{\circ} \mathrm{C}$. In the initial cycle $\left(60^{\circ} \mathrm{C}\right)$, the overall shapes of the potential profiles were identical, although the degree of polarization was higher in the cells cycled at $60^{\circ} \mathrm{C}$ (Fig. 4a). As the number of cycles increased, larger differences were observed in the degree of polarization, with more rapid fading of the cells without TMSPO than those with TMSPO. As a result, the cells cycled with TMSPO displayed outstanding retention $(82.0 \%)$, whereas those cycled without TMSPO showed only $64.6 \%$ retention after 50 cycles (Fig. 4b). These results indicate that the addition of TMSPO to the cell improved the cycle life of the NCM811 electrode.

To investigate the role of TMSPO in the cell, the cycled NCM811 electrode was recovered, and its surface morphology was characterized using SEM (Figs. $5 \mathrm{a}$ and $5 \mathrm{~b}$ ). The surface morphology of the recovered NCM811 electrode cycled with TMSPO was relatively clean. In contrast, decomposed adducts caused by electrolyte decomposition covered the surface of the recovered NCM811 electrode cycled with only the standard electrolyte, consistent with the difference in cycling retention, as shown in Fig. 4. The EIS results of the cycled electrodes supported these behaviors (Fig. 6). In the initial cycle, the cell cycled with TMSPO exhibited lower $\mathrm{R}_{\mathrm{SEI}}(11.1 \Omega)$ than that cycled with only the standard electrolyte $\left(\mathrm{R}_{\mathrm{SEI}}\right.$ : $29.0 \Omega$ ). After 10 cycles, the internal resistances 

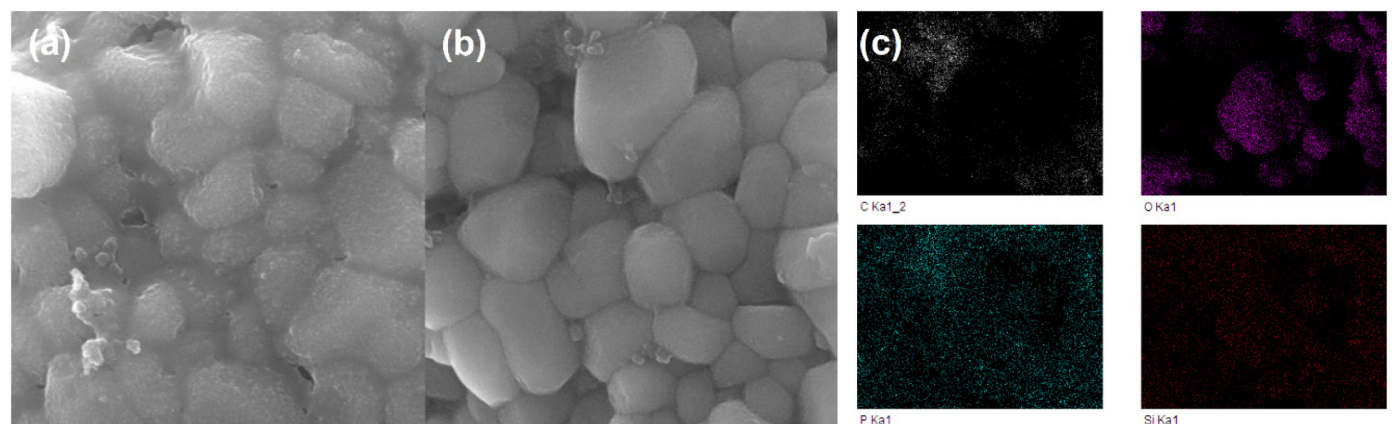

Fig. 5. SEM images of recovered NCM811 electrodes cycled with (a) standard electrolyte and (b) TMSPO-containing electrolyte after 50 cycles. (c) EDS analysis of an NCM811 electrode cycled with TMSPO-containing electrolyte.
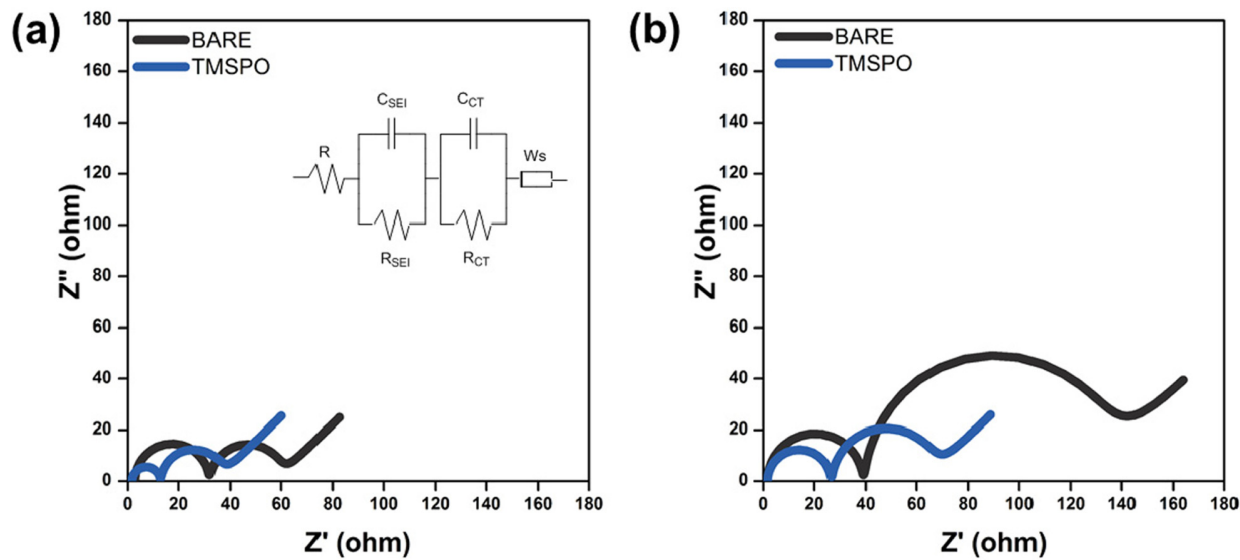

Fig. 6. EIS analysis of an NCM811 electrode (charged to $4.3 \mathrm{~V}$, vs. $\mathrm{Li} / \mathrm{Li}^{+}$) after (a) 1 cycle and (b) 10 cycles (black: standard electrolyte; blue: standard electrolyte $+2 \%$ TMSPO)

increased significantly in the NCM811 electrode cycled without TMSPO $\left(\mathrm{R}_{\mathrm{SEI}}: 36.9 \Omega\right.$ and $\mathrm{R}_{\mathrm{CT}}$ : $87.6 \Omega)$, whereas the NCM811 electrode cycled with TMSPO exhibited lower resistances $\left(\mathrm{R}_{\mathrm{SEI}}: 24.5 \Omega\right.$ and $\mathrm{R}_{\mathrm{CT}}: 36.8 \Omega$ ). These results imply that the use of TMSPO effectively enhanced the surface stability of the NCM811 electrode.

Further energy-dispersive X-ray spectroscopy (EDS) studies of the effect of TMSPO on the NCM811 cathode revealed C, O, P, and Si (i.e., the major elements that constitute TMSPO) on the NCM811 electrode cycled with TMSPO (Fig. 5c). This finding provided evidence of CEI layer formation on the NCM811 electrode surface. FT-IR analyses provided additional useful information to explain the formation of these layers (Fig. 7). All of the recovered NCM811 electrodes without TMSPO showed intrinsic signals typical of those observed for decomposition adducts from the main solvents

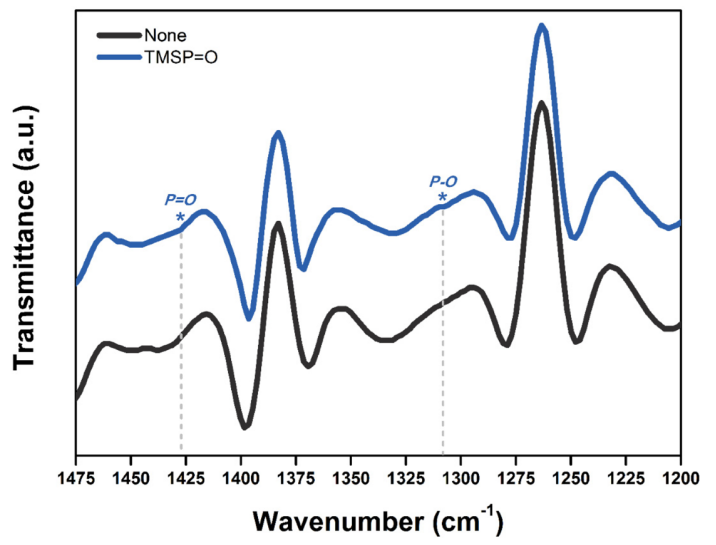

Fig. 7. FT-IR analysis of recovered NCM811 electrodes cycled with (a) standard electrolyte and (b) TMSPOcontaining electrolyte.

(cyclic and acyclic carbonate) in Li salt $\left(\mathrm{LiPF}_{6}\right)$ $[16,26]$. In addition, the recovered NCM811 elec- 
(a)

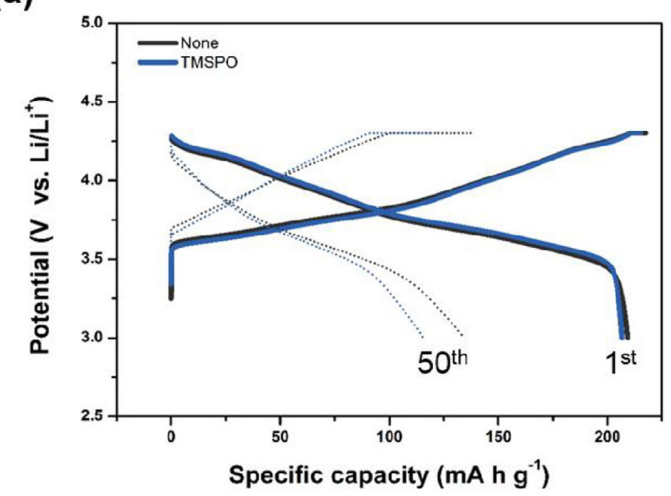

(b)

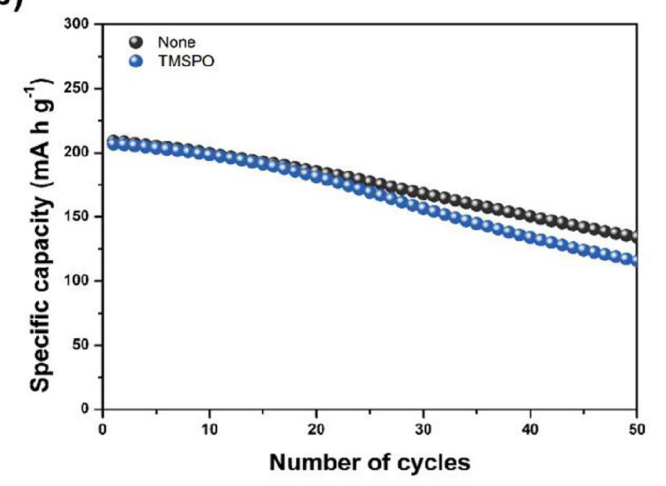

Fig. 8. Electrochemical performances of cells cycled with standard electrolyte (black) and TMSPO-containing electrolyte (blue): (a) potential profiles of the initial cycle (line) and 50 cycles (dot) and (b) cycling retentions. All cycles were performed at $60^{\circ} \mathrm{C}$.

trodes cycled with TMSPO exhibited distinct transmittance signals at 1425 and $1307 \mathrm{~cm}^{-1}$, which suggest the existence of phosphate functional groups [27]. These findings strongly indicated that the electrochemical oxidation of TMSPO resulted in the formation of phosphate-based CEI layers on the surface of the NCM811 electrode, thereby enhancing the interfacial stability and cycling performance, even at high temperatures.

Finally, we demonstrated the effect of the temperature at which the CEI layer formed on the electrochemical performance (Fig. 8). The temperature at which CEI layer formation occurred was changed from room temperature to $60^{\circ} \mathrm{C}$, and the cycling behavior was monitored. Interestingly, the electrochemical behavior of the cell precycled at $60^{\circ} \mathrm{C}$ was quite different from that of the cells precycled at room temperature. In terms of the potential profiles, the polarization ability of the cells cycled with TMSPO was high, even exceeding that of the cells cycled without TMSPO in the initial cycle (Fig. 8a). In addition, the cycling retention of the cell was differed substantially from the aforementioned results, with serious capacity fading $(56.0 \%)$ observed in the cells cycled with TMSPO. The specific retention capacity of the cells cycled without TMSPO was similar $(64.2 \%)$ to that of the cells precycled at room temperature $(64.6 \%)$. Thus, when the cells were exposed to a high temperature before the formation of the CEI layer on the NCM811 electrode, additional fading $(26.0 \%)$ occurred and was accelerated in the cells with TMSPO. These findings demonstrate that the temperature at which the CEI layer formed is a crucial factor in determining the overall electrochemical performance of the cells. The role of the temperature might be explained by differences in the growth rate of the CEI layer on electrode surface. Although the growth rate of the CEI layer was slow at low temperature, wide CEI coverage of the surface of the NCM811 electrode was achieved, resulting in the formation of a uniform CEI layer on the electrode surface. Preliminary studies suggested that precycling cells at a low temperature generally affected their cycling performance [28,29]. According to these results, to utilize TMSPO efficiently, the formation of the CEI layer should likely occur at a low temperature.

\section{Conclusion}

To enhance the high-temperature performance of high-Ni NCM cathodes, bi-functionalized TMSPO was selected as an additive, and its electrochemical performance was evaluated. TMSPO aided the formation of phosphate-based CEI layers on the surface of NCM811 electrodes via an electrochemical oxidation reaction during the initial formation step and effectively improved interfacial stability by suppressing electrolyte decomposition. As a result, the cells containing TMSPO exhibited enhanced cycling retention $(82.0 \%)$ at a high temperature after 50 cycles. In addition, the temperature at which the CEI layer formed played a key role in determining its effectiveness. To produce a more effective CEI layer 
on an NCM811 electrode, the formation process should occur at a low temperature

\section{Acknowledgments}

This work was supported by Incheon National University Research Grant in 2016 (20161986).

\section{References}

[1] B. Scrosati, J. Hassoun, Y.-K. Sun, Energy Environ. Sci. 2011, 4, 3287-3295.

[2] A. Yoshino, Angew. Chem. Int. Ed. 2012, 51, 57985800 .

[3] J. W. Fergus, J. Power Sources 2010, 195, 939-954.

[4] T.-H. Kim, J.-S. Park, S. K. Chang, S. Choi, J. H. Ryu, H.-K. Song, Adv. Energy Mater. 2012, 2, 860-872.

[5] M. M. Thackeray, C. Wolverton, E. D. Isaacs, Energy Environ. Sci. 2012, 5, 7854-7863.

[6] M. Armand, J.-M. Tarascon, Nature, 2008, 451, 652657.

[7] D.-J. Lee, B. Scrosati, Y.-K. Sun, J. Power Sources 2011, 196, 7742-7746.

[8] S.-H. Lee, C. S. Yoon, K. Amine, Y.-K. Sun, J. Power Sources, 2013, 234, 201-207.

[9] W. Cho, S.-M. Kim, K.-W. Lee, J. H. Song, Y. N. Jo, T. Yim, H. Kim, J.-S. Kim, Y.-J. Kim, Electrochim. Acta 2016, 198, 77-83.

[10] J. M. Park, D. Kim, H. B. Kim, J. H. Bae, Y.-J. Lee, J. I. Myoung, E. Hwang, T. Yim, J. H. Song, J.-S. Yu, J. H. Ryu, J. Korean Electrochem. Soc. 2016, 19, 80-86.

[11] S.-J. Yoon, S.-T. Myung, Y.-K. Sun, J. Electrochem. Soc. 2014, 161, A1514-A1520.

[12] B.-B. Lim, S.-T. Myung, C. S. Yoon, Y.-K. Sun, $A C S$ Energy Lett. 2016, 1, 283-289.

[13] W. Liu, P. Oh, X. Liu, M.-J. Lee, W. Cho, S. Chae, Y. Kim, J. Cho, Angew. Chem. Int. Ed. 2015, 54, 44404457.
[14] Y. Koyama, H. Arai, I. Tanaka, Y. Uchimoto, Z. Ogumi, Chem. Mater. 2012, 24, 3886-3894.

[15] H. J. Yu, Y. M. Qian, M. R. Otani, D. M. Tang, S. H. Guo, Y. B. Zhu, H. S. Zhou, Energy Environ. Sci. 2014, 7, 1068-1078.

[16] K. S. Kang, S. Choi, J. Song, S.-G. Woo, Y. N. Jo, J. Choi, T. Yim, J.-S. Yu, Y.-J. Kim, J. Power Sources 2014, 253, 48-54.

[17] T. Yim, K. S. Kang, J. Mun, S. H. Lim, S.-G. Woo, K. J. Kim, M.-S. Park, W. Cho, J. H. Song, Y.-K. Han, J.-S. Yu, Y.-J. Kim, J. Power Sources 2016, 302, 431-438.

[18] Y.-M. Song, C.-K. Kim, K.-E. Kim, S. Y. Hong, N.-S. Choi, J. Power Sources 2016, 302, 22-30.

[19] B. Koo, J. Lee, Y. Lee, J. K. Kim, N.-S. Choi, Electrochim. Acta 2015, 173, 750-756.

[20] Y.-K. Han, J. Yoo, T. Yim, Electrochim. Acta 2016, 215, 455-465.

[21] Y.-K. Han, J. Yoo, T. Yim, J. Mater. Chem. A 2015, 3, 10900-10909.

[22] T. Yim, S.-G. Woo, S. H. Lim, W. Cho, J. H. Song, Y.K. Han, Y.-J. Kim, J. Mater. Chem. A, 2015, 3, 61576167.

[23] N. N. Sinha, J. C. Burns, J. R. Dahn, J. Electrochem. Soc. 2014, 161, A1084-A1089.

[24] J. Zhang, J. Wang, J. Yang, Y. NuLi, Electrochim. Acta 2014, 117, 99-104.

[25] G. Yan, X. Li, Z. Wang, H. Guo, C. Wang, J. Power Sources 2014, 248, 1306-1311.

[26] T. Yim, S. H. Kim, S.-G. Woo, K. Lee, J. H. Song, W. Cho, K. J. Kim, J.-S. Kim, Y.-J. Kim, RSC Adv. 2014, 4, 19172-19176.

[27] E. Pretsch, P. Buhlmann, C. Affolter, Structure Determination of Organic Compounds, Springer, Germany, 2000.

[28] J. Vetter, P. Novák, M. R. Wagner, C. Veit, K.-C. Möller, J. O. Besenhard, M. Winter, M. Wohlfahrt-Mehrens, C. Vogler, A. Hammouche, J. Power Sources 2005, 147, 269-281.

[29] G. Sarre, P. Blanchard, M. Broussely, J. Power Sources 2004, 127, 65-71. 